\title{
Genos, chōra et guerre dans le prologue du Timée-Critias
}

\author{
Tanja Ruben
}

\begin{abstract}
This article defends the claim that Critias' discourse in the prologue of the Timaeus (2od-26e) introduces not only his own discourse about Atlantis in the Critias, but also that of Timaeus on the origin of the cosmos and the human being. In both cases, the concepts of genos ("family," "genus," "species"), chōra ("territory"), and war play a role. There are thus thematic links between the two discourses: the genealogies, the description of the territory of Ancient Athens, and the evocation of its war against Atlantis are taken up and transposed to the cosmic level in Timaeus' discourse, especially in the second part, where he describes the genesis of the four kinds of perceptible particles in the chöra (48a-68d).
\end{abstract}

\section{Keywords}

Plato - Timaeus - Critias - genealogy - chōra - receptacle

Le philologue ou l'historien qui lit le début du Timée et le Critias parce qu'il s'intéresse au récit intrigant de l'Atlantide et à ses modèles littéraires ou historiques se heurte inévitablement au problème suivant: pour raconter la meilleure cité en guerre, pourquoi est-il nécessaire de commencer par le commencement, et d'exposer d'abord sur plus de 65 pages de l'édition Estienne la naissance du cosmos et des hommes, avant de relater enfin, et pas même jusqu'au bout, la guerre athéno-atlante ? Quelle est la fonction du discours cosmogonique et anthropogonique de Timée dans le plan annoncé par Critias à la fin du prologue ? Serait-il impossible de prononcer un discours sur la guerre de la meilleure cité sans celui de Timée?

La question se pose dans l'autre sens au philosophe qui prend la peine de lire d'abord le long prologue imbriqué avant de se lancer dans le discours « philosophique » de Timée : si Platon avait l'intention d'exposer sa conception du 
cosmos et de la constitution psychique et physique de l'homme au moyen d'un récit cosmogonique et anthropogonique, pourquoi l'a-t-il encadré par un récit de guerre (inachevé) entre deux cités «mythiques»? Le prologue du Timée serait-il davantage qu'un prologue au Critias? Si oui, qu'apporte-il de plus à la compréhension du discours de Timée sur le cosmos et sur l'homme?

Ces deux questions sont évidemment liées : d'une part, du point de vue du projet discursif, le discours de Timée devrait préparer celui de Critias le jeune. Comme ce dernier l'explique lui-même en présentant à Socrate le «menu » du festin de paroles, Timée est censé lui fournir les êtres humains, nés dans un cosmos nouveau, pour peupler l'ancienne Athènes, qui est la « vraie » Athènes primordiale, désormais identifiée à la meilleure cité (27a2-b6). La cosmogonie et l'anthropogonie exposées par Timée ont leur fin dans la politogonie qui est l'objet du discours de Critias. A cet égard, le discours de Timée forme un long prélude - comparable aux Hymnes homériques ou à la Théogonie - au discours d'inspiration iliadique de Critias. D'autre part, si on lit le Timée-Critias comme une unité dialogique, le discours de Timée se trouve au centre. Or, comme dans la République, c'est au centre qu'apparaissent les concepts philosophiques les plus novateurs, même si, du point de vue discursif, la partie centrale n'est qu'une digression. Si donc le prologue du Timée-Critias est vraiment un prologue, il n'introduit pas seulement les personnages mais aussi la thématique de la suite dialogique tout entière.

C'est pourquoi il me semble intéressant et important d'examiner en quoi le prologue du Timée-Critias prépare et éclaire aussi le discours de Timée. Ainsi peut-on montrer que la cité récapitulée par Socrate au début du prologue (17c1-19b2) ne rappelle pas seulement au lecteur la kallipolis de la République, mais qu'elle anticipe également la description de l'homme dans le discours de Timée (en particulier $69 \mathrm{c}^{-}-72 \mathrm{~d}_{3}$ ). L'homme y apparaît en effet, du point de vue de sa constitution physique et psychique, comme une cité en miniature :Timée réduit de nouveau en petits caractères la cité que Socrate, dans la République, avait fait naître en paroles pour examiner en gros caractères la justice humaine $(368 c 7-369 b 4)$. Ici, je me suis toutefois proposé la tâche plus difficile de trouver des liens thématiques entre le récit de Critias dans le prologue et le discours de Timée qui lui fait suite. Ceux que j’ai repérés se tissent autour de trois mots clés : chōra («terre civique», «territoire»), genos («famille», «genre » ou « espèce ») et guerre.

\subsection{Le vou de Socrate}

Louverture du Timée-Critias inscrit les discours qui y sont prononcés dans le cadre d'une hospitalité discursive : Timée, Critias et Hermocrate sont invités à rendre à Socrate un don sous la forme d'un discours équivalant au discours que 
celui-ci leur avait offert la veille. En guise d'aide-mémoire, Socrate récapitule brièvement son discours qui portait sur la meilleure cité et le genre d'hommes devant la constituer. Suit une comparaison par laquelle il essaie d'expliquer à ses trois hôtes ce qu'il éprouve face à la cité qu'il a parcourue la veille avec eux : sa cité lui donne l'impression de manquer de quelque chose, un peu comme des êtres vivants beaux ( $\zeta \hat{\omega} \alpha \varkappa \alpha \lambda \dot{\alpha})$ mais immobiles nous donneraient envie de les voir bouger, plus exactement de les voir exécuter une lutte athlétique $(19 \mathrm{~b} 4-\mathrm{cl})$. La comparaison permet à Socrate de formuler son vœu pour la suite du festin: de même que, face à de beaux êtres vivants immobiles, on a envie de les voir bouger et lutter, de même il aimerait voir la meilleure cité en pleine guerre :

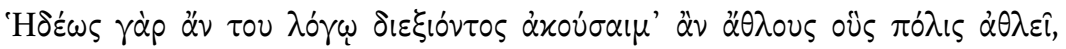

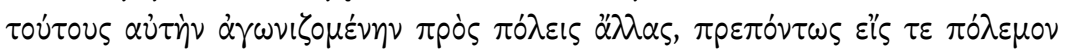

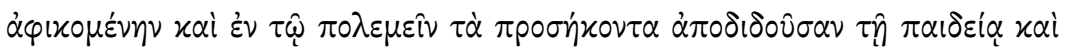

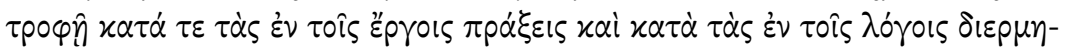

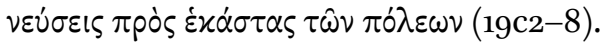

J'aimerais entendre quelqu'un raconter que ces luttes que soutient une cité, elle les affronte, elle aussi, contre d'autres cités, en entrant en guerre comme il faut et en se montrant, pendant la guerre, digne de la façon dont elle a été éduquée et élevée, aussi bien en pratique dans ses actes qu'en paroles dans ses négociations avec chacune des cités ${ }^{1}$.

Socrate souhaite donc que ses trois hôtes prononcent pour lui un discours qui montre la meilleure cité en guerre, de telle sorte quà travers ses actions et ses discours, elle fasse valoir sa belle éducation. La meilleure cité a en effet été créée en paroles pour exceller dans la guerre. Les lignes suivantes $\left(19 \mathrm{~d} 3^{-} \mathrm{e} 8\right)$ suggèrent que Socrate s'attend à un discours mimétique qui, d'un point de vue formel, rappelle l'Iliade avec son alternance de discours et d'actions héroïques, sauf que les héros ne sont désormais plus un Achille ou un Hector mais les gardiens de la meilleure cité et leurs commandants. Socrate a attribué à ces

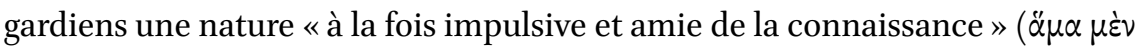
Ө $\mu \circ \varepsilon ı \delta \hat{\eta}, \ddot{\alpha} \mu \alpha \delta \dot{\varepsilon} \varphi\left(\lambda \sigma^{\circ} \sigma \circ \varphi \circ v, 18 \mathrm{a} 5\right)$, puis a décrit leur éducation et leur genre de vie (18a9-19a5). Il se montre convaincu que Timée, Critias et Hermocrate - qui sont à la fois philosophes et politiques, puisqu'ils partagent la même nature et

1 Mes traductions des passages grecs s'inspirent de celles de Rivaud, Platon. CEuvres complètes, tome x, Timée-Critias, et de Brisson, Platon. Timée. Critias. 
la même éducation que les héros qu'ils sont censés représenter - sont les seuls capables d'offrir un tel discours guerrier (19e8-2ob7).

Avant d'examiner ce que propose Critias pour répondre au vœu de Socrate, il convient de rappeler brièvement comment naît la guerre dans la République. On comprendra ainsi mieux quel rôle jouent la chōra et la naissance d'un genos humain dans la réalisation de ce vou.

\subsection{La naissance de la guerre dans la République}

La guerre apparaît au livre II, au moment où l'on passe d'une cité frugale et saine à une cité malade qui vit dans le luxe (372a-373e). La cité que Socrate et Glaucon viennent de construire en paroles est née du constat que la division du travail permet plus aisément aux êtres humains de pourvoir à leurs besoins de nourriture, de vêtements et d'abri (369b5-371e11). Les hommes et les femmes y mènent une vie modeste et se contentent d'une nourriture végétarienne, faite de pain et de galettes accompagnés de sel, d'olives, de fromage, d'oignons et de légumes bouillis, et de figues, de pois chiches, de fèves, de baies de myrtes et de glands rôtis au feu comme dessert (372a5-372d3). Selon Glaucon, une telle cité rurale, qui se nourrit de glands, couchée sur un lit de feuilles, fait penser à «une cité de pourceaux» ( $\dot{v} \omega \hat{\omega} \pi \dot{\lambda} \lambda\left(v, 372 \mathrm{~d}_{4}\right)$. Dans une cité humaine, on s'attendrait, selon lui, à une certaine qualité de vie, comprenant notamment une nourriture plus variée, avec de la viande et de vrais desserts ainsi qu'une culture de table digne de ce nom ( $372 \mathrm{~d} 7$-e1). Socrate accepte de modifier son tableau et de laisser la cité saine qu'il vient de construire pour une cité luxueuse ( $\tau \rho \nu \varphi \hat{\omega} \sigma \alpha \nu \pi \dot{\lambda} \lambda \mathrm{c} v, 372 \mathrm{e} 3)$ qu' « il faut donc remplir d'une

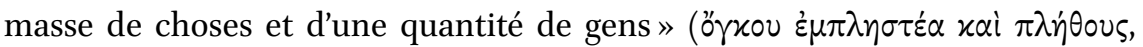
$\left.373 \mathrm{~b}_{3}-4\right)$ : lits, tables et autres meubles, viandes, desserts, parfums, hétaïres, peinture et broderie, or et ivoire; puis chasseurs, artistes et artisans de toutes sortes, ou encore pédagogues, nourrices, cuisiniers, bouchers, porchers et autres serviteurs $\left(373^{\mathrm{a}-\mathrm{c})}\right)$. Cependant, une cité qui dépasse la limite des nécessités vitales pour se livrer à l'acquisition illimitée de richesses (

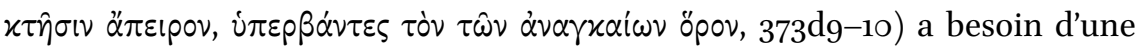
chōra (le terme apparaît en $373 \mathrm{~d} 4$ ) plus étendue pour faire paître son bétail et semer des céréales. C'est pourquoi elle commence à transgresser ses frontières et à retrancher des parcelles de territoire appartenant aux cités voisines. La cité luxueuse fait la guerre pour étendre sa chōra, qui est comme son corps, et, ce faisant, enfle $(\varphi \lambda \varepsilon \gamma \mu \alpha i v o v \sigma \alpha \nu \pi \dot{\lambda} \lambda \iota v, 372 \mathrm{e} 8)^{2}$. L'examen de ce passage de

2 Pour une analyse détaillée des genres de vie de la cité de pourceaux et de la cité luxueuse dans la République II 372a-373e, voir Campese et Canino, «La genesi della polis », chap. II «La città dei maiali » (307-317) et chap. III « Polis tryphosa » (318-332). 
la République nous apprend que la guerre naît donc au moment où une cité essaie d'élargir sa chōra aux frais d'une autre cité. Par conséquent, pour revenir au prologue du Timée, qui veut répondre au vœu de Socrate et montrer la meilleure cité en train de faire la guerre devra lui donner une chōra et définir les limites de celle-ci par rapport aux chōrai des cités voisines. C'est seulement ainsi qu'elle pourra entrer dans une guerre (défensive) contre une autre cité3.

Par ailleurs, si l'on prend au sérieux la remarque de Glaucon sur la cité de pourceaux, des deux cités - saine ou luxueuse - seule la dernière est vraiment humaine ${ }^{4}$. Sous certains aspects, en effet, la vie paisible et saine des hommes et des femmes de la première cité de Socrate rappelle celle des êtres humains sous le règne de Cronos dans le récit du Politique $(271 \mathrm{~d}-\mathrm{e})$ : chacune des espèces vivantes, dont les êtres humains, formait alors un troupeau gardé séparément par une divinité, si bien qu'il n'y avait ni guerre $(\pi \dot{\lambda} \lambda \varepsilon \mu \circ \varsigma)$ ni guerre civile $(\sigma \tau \dot{\alpha}-$ $\sigma(\zeta)^{5}$. Ici, comme dans la République, les humains-animaux se distinguent des êtres humains actuels par l'absence d'activité belliqueuse. Or la guerre est aussi ce qui fait la différence entre les humains et les dieux; car on a tort de croire que les dieux luttent et se font la guerre, comme Socrate l'explique un peu plus loin quand il entreprend de censurer les récits d'Homère et d'Hésiode (378b8e3, cf. aussi Critias 1ogb1-5). La guerre apparaît seulement avec le genre (үદ́vos) humain: la genèse de la guerre et celle des êtres humains vont de pair. Avec l'entrée en guerre de la cité construite en paroles par Socrate commence donc le temps propre au genre humain. Outre le fait qu'il faut pouvoir délimiter la chōra de la meilleure cité et celle(s) de ses adversaires, qui veut la montrer en guerre devra déterminer sa place dans la lignée généalogique des êtres vivants. Comme on le verra à propos du récit de Critias, ces deux exigences sont liées :

3 Bien que la guerre reste un mal qui ne pourra jamais être éradiqué, la cité, selon Platon, devrait viser la paix. C'est pourquoi l'ancienne Athènes (que Critias identifie avec la meilleure cité) excelle dans une guerre défensive contre l'Atlantide, alors que celle-ci, en proie à une envie illimitée de richesses, mène une guerre offensive contre les anciens Athéniens et les autres cités grecques. Sur l'attitude de Platon à l'égard de la guerre (et de la paix), voir Cambiano, «La pace in Platone e in Aristotele», et Brisson, «Platon face à la guerre ».

4 A juste titre, Campese et Canino, «La genesi della polis », 307-308, observent que les hommes de la première cité mènent une vie frugale et rurale mais non sauvage, puisqu'ils se nourrissent d'aliments cuits. Pour être précis, il faudrait donc parler non pas d'une « cité animale » mais d'une « cité d'animaux domestiques» (comme le sont les cochons justement).

5 La notion des pâtres divins en rapport avec la paix apparaît également dans le Critias 1ogb6-7 et dans les Lois IV 713c2-e3. Cf. aussi la vie modérée et paisible que mènent les survivants du déluge dans les montagnes au livre II 678e-679e des Lois. Sur les ambiguïtés de lâge d'or chez Platon, voir Vidal-Naquet, Le chasseur noir. Formes de pensée et formes de société dans le monde grec, $361-380$. Dans le récit du Politique, constate-t-il, « [l] e paradis de l'âge d'or est, en définitive, un paradis animal » (373). 
le discours généalogique est toujours aussi un discours géographique et politique, ou mieux « chôrologique $»^{6}$.

Prononcer un discours sur la meilleure cité en train d'exécuter des mouvements de guerre, comme le souhaite Socrate, signifie donc tenir compte de deux choses qui semblent corrélées: la naissance d'un genos humain (distinct des genē des dieux et des animaux) et la chōra de la cité, c'est-à-dire sa «terre civique » ou son « territoire »(délimité par rapport aux chōrai des cités voisines).

\subsection{La réponse de Critias au vou de Socrate}

Regardons maintenant comment Critias s'y prend dans le prologue du Timée-Critias pour répondre à Socrate. A peine celui-ci a-t-il exprimé son vœu d'entendre un discours montrant la meilleure cité en guerre que Critias se saisit de la parole pour lui présenter, au nom des trois hôtes du jour, «un discours

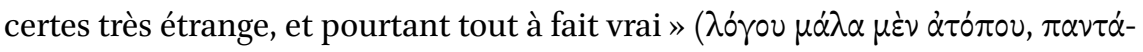

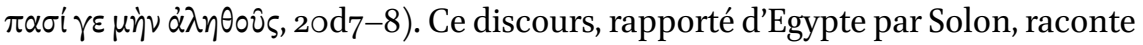
la guerre entre l'ancienne Athènes et l'Atlantide, et, à condition que l'on identifie la meilleure cité avec l'ancienne Athènes, répondrait parfaitement aux attentes de Socrate. C'est du moins ce dont Critias essaie de convaincre Socrate dans le prologue. Son propos présente la structure suivante:

1. La transmission du discours «absolument vrai» sur la guerre athénoatlante à l'intérieur du genos de Critias le jeune (2od7-21d8)

2. Le récit de Critias l'ancien $(21 \mathrm{e}-25 \mathrm{~d} 6)$ racontant le voyage de Solon en Egypte et résumant le discours d'un très vieux prêtre égyptien (22b425d6). Ce dernier peut être divisé en trois parties :

a) La «vraie » généalogie de la cité d'Athènes (22b4-23d1)

b) La production du genos des anciens Athéniens dans la chōra attique et son organisation politique $(23 \mathrm{~d} 4-24 \mathrm{~d} 6)$

c) La chōra immense des Atlantes dans «la vraie mer» et la guerre qu'ils ont menée contre les anciens Athéniens, suivie de leur défaite et de leur disparition $(24 \mathrm{~d} 6-25 \mathrm{~d} 6)$

6 Graf, «Zwischen Autochthonie und Immigration: Die Herkunft von Völkern in der Alten Welt», 71, remarque à propos du récit généalogique des descendants de Pyrrha et de Deucalion, transmis dans le Catalogue des femmes : «Die Analyse zeigt, was dieser Mythos von den Ahnen der griechischen Stämme leistet. Er ist ein Mittel, die Geographie des griechischen Festlandes zu denken. In einer Epoche, in der es keine Karten gibt und keine schriftlichen Schematismen, mit denen man etwas derart Kompliziertes wie die vielen Völker Griechenlands ordnen kann, zeichnet er eine völkergeographische Karte ». De même Fowler, «Genealogical Thinking, Hesiod's Catalogue, and the Creation of the Hellenes », 1 : «For those within the system a genealogy is a map. They can read its signs. To the names are attached stories, thousands of them ; collectively they gave the listeners their sense of history and their place in the world». 
3. La remémoration de l'ancien discours par Critias le jeune $\left(25 \mathrm{~d}_{7}-26 \mathrm{c}_{5}\right)$ et sa proposition de transposer la meilleure cité de Socrate « dans la réalité » en l'identifiant avec l'ancienne Athènes $\left(26 c_{5}-26 \mathrm{e} 1\right)$

On peut constater que les généalogies et les descriptions géographiques et territoriales liées aux termes grecs de genos et de chōra jouent un rôle important tout au long du propos de Critias. La nouveauté ici, par rapport au passage du livre II de la République, c'est le problème de la «vérité » ou de la «réalité » $(\alpha \lambda \hat{\eta} \theta \varepsilon ı \alpha)$, qui surgit là seulement au livre III en rapport avec le «noble mensonge » racontant la naissance, de la chōra, d'un corps de guerriers parfaits $(414 \mathrm{~b} 8-415 \mathrm{~d} 2)$.

Dans ce qui suit, j'examinerai d'abord le rôle des généalogies et des descriptions « chôrologiques » et leur rapport avec la guerre dans le discours du prêtre égyptien (22b4-25d6). J'essaierai ensuite de montrer en quoi ce discours prépare le deuxième discours de Timée $(48 \mathrm{a} 7-68 \mathrm{~d} 7)$, consacré à la genèse de corpuscules perceptibles qui se déroule dans la chōra, cette troisième espèce d'être que le philosophe de Locres est contraint d'introduire (cf. 48e2-49a6, 52a8$5^{2 \mathrm{~d}} 4$ ). Cela me permettra de comparer, dans une troisième partie, les combats guerriers de ces corpuscules à ceux entre l'ancienne Athènes et l'Atlantide. Pour finir, j'aimerais soulever la question de la vérité en rapport avec les discours généalogiques et « chôrologiques » du prêtre égyptien et de Timée.

Afin d'inciter les prêtres de Saïs à parler de l'origine de leur cité ( $\pi \varepsilon p \grave{~} \tau \hat{\omega} \nu$ àp$\chi \alpha i \omega \nu, 22 a 4-5)$, l'Athénien Solon tient ce que l'on pourrait appeler un discours « archéo-mytho-généalogique » : il cherche à remonter jusqu'aux premiers ancêtres panhelléniques et même humains de sa propre cité, Deucalion et Pyrrha, puis, avant le déluge, Niobé et Phorôneus, que l'on appelle le premier homme, et il tente de calculer leurs années de vie (22a4-b3). Le prêtre lui répond à son tour par un discours généalogique, qui a pour but de lui révéler la « véritable » origine ( $\alpha p \chi \eta ́)$ de sa cité, Athènes. Il se moque gentiment du discours de Solon,

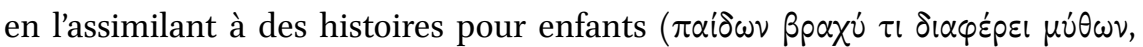
$23 b_{5}$ ) que se raconte un peuple à peine alphabétisé, pour enchérir aussitôt, en repoussant la «véritable » origine des Athéniens bien plus haut dans le temps, avant d'autres déluges qui auraient précédé celui de Deucalion, le seul connu des Grecs. Plus loin, il la situe exactement 9000 ans auparavant (cf. 23e4-5). C'est à cette époque qu'aurait vécu en Attique même, non encore ravagée par les déluges, sinon le premier genre humain, en tout cas le plus beau et le meilleur qui ait jamais vu le jour : 


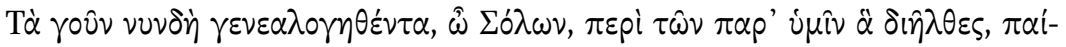

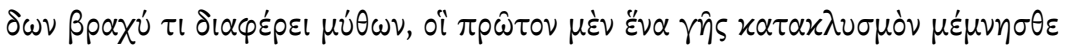

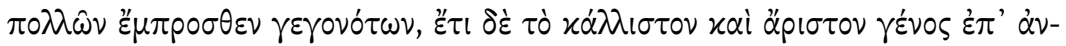

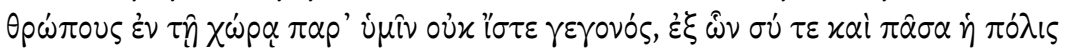

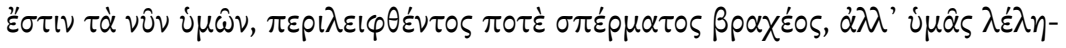

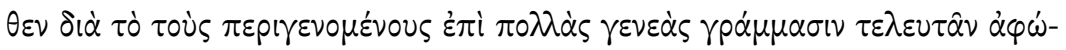
vous (23b3-c3).

En tout cas, les généalogies concernant les gens de chez vous que tu viens, Solon, de passer en revue, diffèrent bien peu des récits des enfants. Car d'abord, vous ne gardez le souvenir que d'une seule submersion de la terre, alors qu'il y en a eu beaucoup auparavant. En outre, le plus beau et le meilleur genre (genos) du temps des hommes, vous ignorez qu'il est né chez vous, dans votre territoire (chōra); c'est d'eux que vous descendez, toi et l'ensemble de la cité qui est aujourd'hui la vôtre, parce que jadis un peu de semence s'en est conservée. Mais vous en avez perdu le souvenir parce que, pendant de nombreuses générations, les survivants sont morts sans avoir fait entendre leur voix à travers des écrits.

Notons que l'identification des citoyens de la meilleure cité de Socrate avec ce genos humain exceptionnel, que Critias le jeune propose tout à la fin $(26 \mathrm{c} 7-26 \mathrm{~d} 5)$, ne les prive pas de leur nature paradigmatique, mais les rend humains : d'êtres vivants $(\zeta \hat{\omega} \alpha)$ - beaux mais immobiles, pour reprendre les mots de Socrate - les citoyens de la meilleure cité deviendront « le plus beau et le meilleur genos du

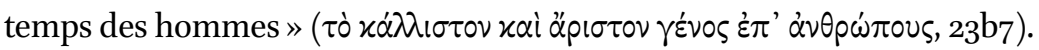

Dansla deuxième partie de son discours, le vieux prêtre explique que ce genre humain excellent est le produit commun de trois divinités : Gê, Héphaïstos et surtout Athéna (23d6-e2). Celle-ci a recueilli de Gê et d'Héphaïstos le rejeton ( $\tau i$ $\sigma \pi \varepsilon \dot{p} p \mu \alpha, 23 \mathrm{e} 1)$ de la future cité d'Athènes et l'a nourri ( $\left.\varepsilon^{\prime} \theta \rho \varepsilon \psi \varepsilon v, 23 \mathrm{~d} 7\right)$ et éduqué (દ̇ $\pi \alpha i \delta \varepsilon v \sigma \varepsilon v, 23 \mathrm{~d} 7)$. En réalité, le prêtre ne révèle ici à Solon (l'Athénien!) rien d'autre que le récit sacré (izpòs $\lambda o ́ \gamma o \varsigma$ ) du festival des Panathénées, en marge duquel Socrate rencontre Timée, Critias et Hermocrate (cf. 21a2-3 et 26e3-4). Ce récit raconte la naissance d'Erichthonios (ou d'Erechthée), l'enfant de Gê, fécondée par Héphaïstos épris d'Athéna, et son éducation par la déesse tutélaire de la cité7.

7 La version traditionnelle de ce récit se retrouve entre autres dans Hom. Il. II, 546-551; Eur. Ion 266-274 et Ps.-Apollod. III, 14, 6. Voir à ce propos aussi Parker, « Myths of Early Athens », surtout 193-197, et Bonnard, Le Complexe de Zeus. Représentations de la paternité en Grèce ancienne, 81-88 (avec de nombreuses indications bibliographiques). 
Léducation qu'Athéna a donnée à ce nouvel être est sa constitution politique $(\pi \circ \lambda i \tau \varepsilon i \alpha)^{8}$. Le prêtre poursuit en effet en décrivant l'organisation politique de l'ancienne Athènes au moyen de lois (vópovs, 23e5 et 24a2) qu'il induit à partir de celles encore en vigueur à Saïs, la cité-sœur de l'ancienne Athènes. Ces lois prescrivent une séparation stricte des différents groupes socio-politiques:1) le genos des prêtres, 2) le genos des artisans, des bergers, des chasseurs et des paysans, et enfin 3) le genos des combattants hoplitiques (24a4-24b7). Cette division en trois genē fonctionnels rappelle, bien sûr, celle de la meilleure cité de Socrate, même si celle-ci ne connaît pas de genos de prêtres spécialisés en astronomie (ou bien en astrologie ?), mantique et médecine $\left(24 b_{7}-c_{3}\right)^{9}$. Le prêtre clôt ainsi sa description de l'organisation politique de l'ancienne Athènes :

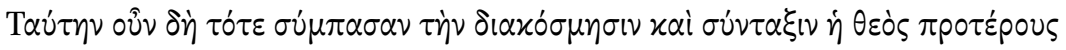

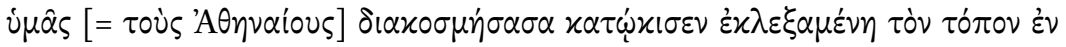

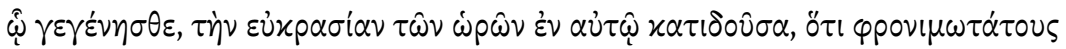

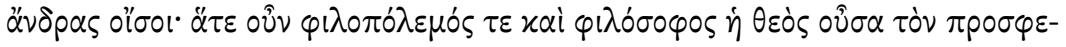

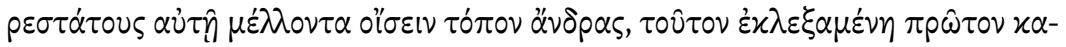

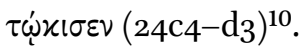

C'est donc alors, après vous [= les Athéniens] avoir entièrement divisés et organisés les premiers, que la déesse a choisi et peuplé la région (topos) où vous êtes nés, apercevant que là l'heureux mélange des saisons allait produire les hommes les plus raisonnables. Etant donné que la déesse aime la guerre et le savoir, c'est la région (topos) qui devait produire des hommes lui ressemblant le plus qu'elle a choisie et peuplée d'abord.

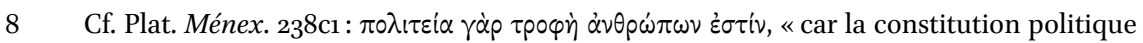
nourrit les êtres humains».

9 Dans son résumé du discours de la veille, Socrate ne mentionne explicitement que deux genē : celui des paysans et des autres arts ( $\tau \dot{\varepsilon} \chi \nu \alpha \iota)$ et celui des défenseurs de la cité (17c6-

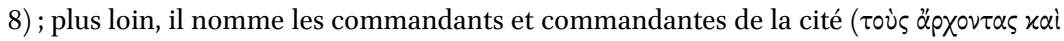

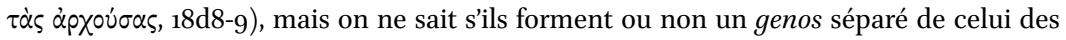
défenseurs.

$10 \mathrm{Vu}$ le contexte, $\delta\left\llcorner\alpha x_{\delta} \sigma \mu \eta \sigma \iota \zeta\right.$ et $\delta ı \alpha \circ \sigma \mu \varepsilon \dot{\omega} \omega$ doivent se référer à la « division » des Saïtiques

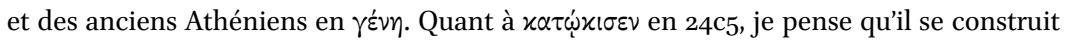

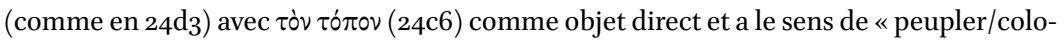
niser la région ». C'est pourquoi et pour mieux mettre en valeur la structure annulaire $(\varkappa \alpha-$

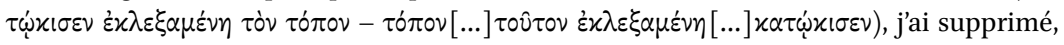
après $x \alpha \tau \dot{\omega} \varkappa ı \sigma \varepsilon v$, la virgule qui se trouve dans l'édition d'Oxford de John Burnet (1902). Pour les différents sens du verbe $\chi \alpha \tau o x i \zeta \omega$, cf. Casevitz, Le vocabulaire de la colonisation

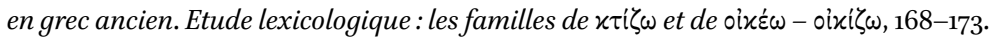


Si le passage 23d6-e2 rappelle le récit sacré des Panathénées, ces lignes-ci évoquent plutôt la version démocratique des rhéteurs, qui vantaient la naissance de l'ensemble des citoyens-soldats athéniens de la chōra même de la cité, qui les aurait nourris et élevés comme une mère et où ils habiteraient depuis toujours en autochtones ${ }^{11}$. Cela ressort plus clairement du passage parallèle du Critias :

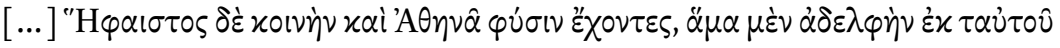

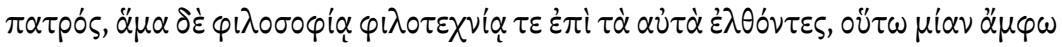

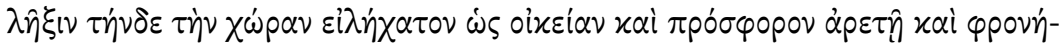

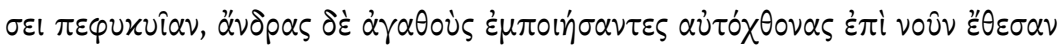
$\tau \grave{\eta} \nu \tau \hat{\jmath} \varsigma \pi 0 \lambda \imath \tau \varepsilon i \alpha \varsigma \tau \alpha^{\prime} \xi \nu(109 \mathrm{c} 6-\mathrm{d} 2)$.

[...] Héphaïstos et Athéna, qui ont un naturel commun, à la fois parce qu'ils sont frère et sœur, issus d'un même père, et parce que l'amour pour le savoir et pour l'art les a orientés dans la même direction, reçurent tous deux en partage pour cette raison un seul lot, ce territoire-ci (chōra), puisqu'il était naturellement approprié et favorable à l'excellence et à la raison, et après y avoir produit comme autochtones des hommes bons, ils leur ont mis dans l'esprit l'ordre constitutionnel.

Les deux passages montrent que la beauté et l'excellence guerrière et philosophique du genos athénien ont deux causes. La première est naturelle: la physis du topos, la «région » ${ }^{12}$, et de la chōra, le «territoire » ${ }^{13}$, où le genos athénien a grandi. Comme d'autres dans le Critias (111e1-5, 112e2-6), ces deux

11 Cf. Lys. 2 (Épit.), 17 ; Isocr. 4 (Panég.), 24-25 et 12 (Panath.), 124-125; Dém. 6o (Épit.), 5 et le pastiche dans Plat. Ménex. 237b2-c3 et 237e2-238a5. Voir aussi le noble mensonge dans la République (414b8-415d2), variante platonicienne de cette idéologie athénienne. Pour les deux versions (aristocratique et démocratique) du récit, cf. Loraux, Les enfants d'Athéna. Idées athéniennes sur la citoyenneté et la division des sexes, 35-73. Pour les aspects nourriciers et maternels de la terre civique ( $\chi \dot{\omega} \rho \alpha$, mais aussi $\gamma \hat{\eta}$ et $\chi \theta \dot{\omega} \nu)$ voir Georgoudi, «Gaia/Gê. Entre mythe, culte et idéologie ».

12 Pradeau, «Être quelque part, occuper une place. TОПО $\Sigma$ et X $\Omega$ PA dans le Timée », 376 : «Tóros signifie une région géographique, qualifiée par un trait caractéristique (morphologique ou climatique). Par exemple, une région élevée, ou bien une région du Nord, ou encore un lieu tempéré ».

13 Ibid. 376-377: «X'́p $\alpha$ signifie le territoire ou la région de la cité, d'un peuple. C'est un terme qui désigne une réalité géographique, mais précisée et circonscrite par son appartenance à une unité politique. A la différence de $\tau o ́ \pi \circ \varsigma, ~ \chi \omega ́ p \alpha$ est toujours nommée d'après un sujet (c'est le territoire de la ville d'Athènes, ou le territoire sur lequel vivent les Lydiens)» (italiques de l'auteur). 
passages suggèrent en effet qu'il existe une relation étroite entre d'une part le topos et la chōra et d'autre part l'excellence physique et psychique d'un genos humain, c'est-à-dire sa beauté et sa raison (phronēsis) qui se manifestent dans son amour de la guerre et du savoir. Plus le climat est équilibré et la terre fertile, mieux les hommes «poussent ». A l'inverse, si le climat change et que le territoire perd de sa fertilité, les hommes deviennent moins beaux et moins raisonnables. Ainsi, au cours des neuf millénaires écoulés depuis l'ancienne Athènes, de nombreux déluges ont-ils provoqué l'érosion de la chōra attique, qui ressemble désormais au corps squelettique d'un malade (cf. Critias 111a6-b7), ce qui en dit long sur l'état physique et psychique de la cité au moment où Critias le jeune parle, vraisemblablement au début de la guerre du Péloponnèse (entre 430 et 425$)^{14}$. De l'ancienne chōra plus étendue et plus riche, il ne reste plus que des indices, que Critias énumère patiemment dans le dialogue qui porte son nom $\left(\operatorname{lod}_{4}-112 \mathrm{~d}_{3}\right)^{15}$.

La deuxième cause de la beauté et de l'excellence du genos athénien est ar-

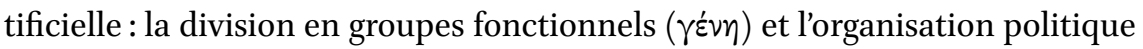

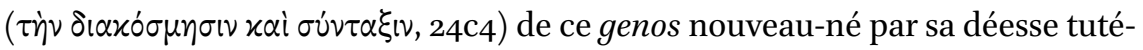
laire, Athéna, qui lui a donné des lois (vómoı). Dans le passage du Critias, il est dit qu'Héphaïstos et Athéna ont introduit dans l'esprit des autochtones athéniens l'ordre constitutionnel ( C'est cette législation qui fait de l'ancienne Athènes «à tout point de vue de

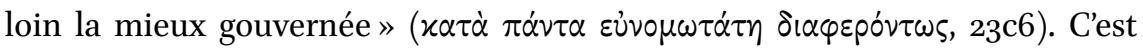
parce qu'elle se distinguait par sa beauté et sa raison naturelles d'une part, par sa perfection constitutionnelle d'autre part, qu'elle a su l'emporter dans la guerre contre l'envahisseur atlante.

Généalogie et « chôrologie » dans le deuxième discours de Timée

Selon le «menu » du festin de paroles que Critias propose à Socrate en 27a2b6, Timée va prononcer un discours qui commence par la genèse du cosmos et

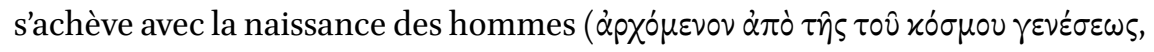

\footnotetext{
14 Date dramatique selon Brisson, Platon. Timée. Critias, 72 et 332.

15 Que les conditions géographiques et climatiques d'un pays déterminent la constitution physique et psychique de ses habitants est une thèse défendue dans le traité hippocratique Des Airs, des Eaux et des Lieux, voir en particulier les chap. 12-13; 15-16 (§ 1-2); 23 (pour l'influence du climat) et 24 (pour celle de la nature du sol) avec la notice de Jouanna, Hippocrate, tome II, $2^{\mathrm{e}}$ partie : Airs, Eaux, Lieux, 6o-64. Cf. aussi Hérodote II 77, 3 et IX 122, 3 ; Platon Lois v 747d-e ; Pradeau, Le Monde de la politique. Sur le récit atlante de Platon, Timée (17-27) et Critias, 248-256.
} 


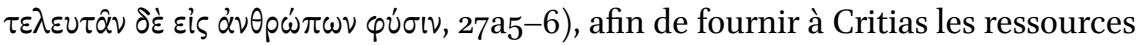
humaines pour peupler l'ancienne Athènes. Il est donc présenté comme un discours généalogique et composé ainsi :

1. Le premier discours (27c1-47e2) qui expose l'œuvre de l'intellect (nous): cosmogonie, théogonie et anthropogonie (première partie)

2. Le deuxième discours qui expose ce qui relève de l'anankê, la «contrainte $\gg(48 \mathrm{a} 7-68 \mathrm{~d} 7)$ :

a) La chōra précosmique remplie d'empreintes sans proportion ni mesure $(48 \mathrm{e} 7-53 \mathrm{a} 8)$

b) La genèse des quatre genē de corpuscules perceptibles et de leurs espèces dans et à partir de la chōra (53b1-68d7)

3. Le troisième discours qui est une combinaison des deux autres (69a6106b7 dans le Critias) : anthropogonie (deuxième partie) et thériogonie La genèse du genre humain s'inscrit dans le projet général du démiurge de fabriquer un monde complet, représentant les idées comprises dans l'idée générique qu'est le vivant intelligible (39e3-40a2). Ces idées sont au nombre de

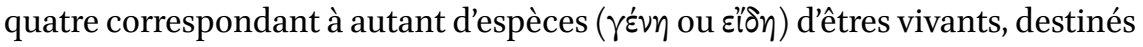
à peupler respectivement le ciel (les dieux), les airs (les oiseaux), les eaux (les poissons et autres animaux aquatiques) et la terre (les animaux pédestres et terrestres). Le vivant intelligible vers lequel l'artisan divin dirige son regard et dont il se sert comme modèle est décrit comme «le plus beau des [êtres

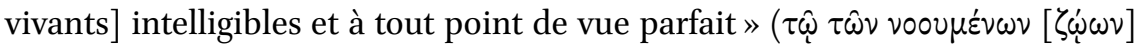

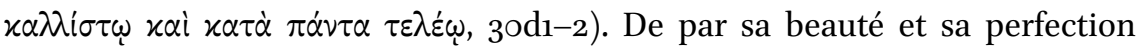
mais aussi de par son immobilité en tant qu'idée stable et inébranlable, ce vivant intelligible rappelle les beaux êtres vivants (zōa kala) immobiles auxquels Socrate a comparé la meilleure cité, exposée la veille en paroles. En tant qu'unité comprenant des genē ou eidē d'êtres vivants différents, il évoque la cité elle-même qui est divisée en genē fonctionnels. De fait, l'artisan divin se trouve face à une tâche similaire à celle que Socrate a confiée à ses trois interlocuteurs : comment mettre en mouvement cet être vivant? Comment passer d'un vivant immobile à un vivant mobile? Plus particulièrement, comment produire un genre humain qui soit capable de se mouvoir et, le cas échéant, de défendre sa cité par la guerre?

Comme pour la meilleure cité, la mise en mouvement de ce vivant intelligible comporte un aspect généalogique et un autre, «chôrologique ». Dans le premier discours de Timée (27c1-47e2), qui explique l'œuvre de l'intellect (vov̂s) divin en faisant abstraction de la contrainte ( $\dot{\alpha} v \alpha \dot{\gamma} \gamma \varkappa \eta)$ et de la troisième espèce d'être qu'est la chōra, l'aspect généalogique est prépondérant. Mais à partir du deuxième discours (48a7ss.), lorsque Timée est forcé de tenir compte en outre des effets de la contrainte dans la genèse du cosmos, les deux aspects 
sont présents conjointement. C'est ce deuxième discours, où entre en jeu la chōra comme nouvelle espèce ontologique, qui m'intéresse ici, car il présente certains parallèles avec le discours du prêtre égyptien. Comme le discours du prêtre, il combine, en effet, les deux aspects : généalogique, car Timée essaie d'expliquer la genèse de corpuscules perceptibles, et "chôrologique », dans la mesure où cette genèse se fait dans un « territoire », qui sert à la fois de réceptacle et de nourrice, la chōra.

Avant la fabrication du cosmos par le démiurge, le feu, l'air, l'eau et la terre

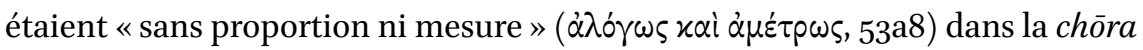
précosmique. Puis, le dieu s'est mis à les organiser:

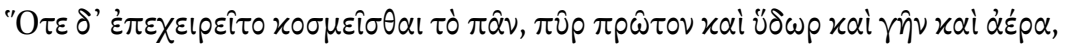

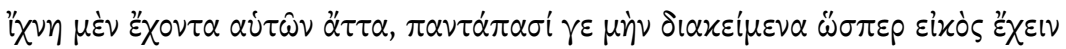

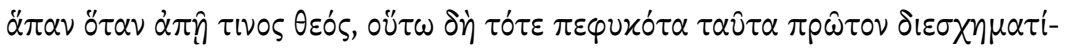

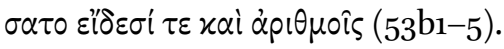

Lorsque l'univers commençait à être ordonné, d'abord le feu, l'eau, la terre et l'air présentaient certes quelques empreintes d'eux-mêmes mais se trouvaient entièrement dans l'état où se trouve vraisemblablement tout lorsqu'un dieu en est absent; ce qui était alors par nature ainsi, il [= le dieu] l'a donc d'abord 'configuré' à l'aide de formes et de nombres.

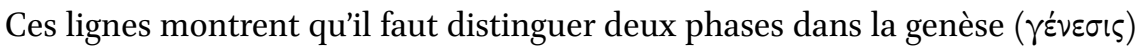
du feu, de l'air, de l'eau et de la terre : d'abord, leur genèse, dans la chōra précos-

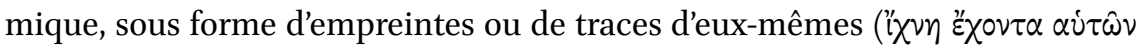
$\left.\alpha^{\prime} \tau \tau \alpha, 53 \mathrm{~b} 2\right)$, c'est-à-dire de la nature ( $\left.\varphi \dot{\sigma} \sigma \varsigma\right)$ et des propriétés $(\pi \dot{\alpha} \theta \eta)$ qu'ils auront plus tard dans le cosmos et qui les rendront perceptibles pour l'homme (devenir traité dans le deuxième proème, en particulier $52 \mathrm{~d}_{4}-53 \mathrm{a} 7$ ). Puis, leur

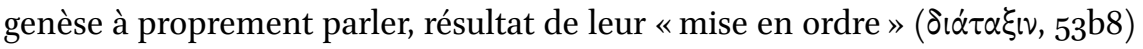

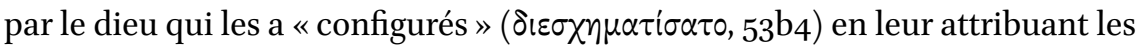
figures géométriques $(\sigma \chi \eta \dot{\eta} \mu \alpha \tau \alpha)$ que sont les quatre polyèdres (thème du deuxième discours, en particulier $53^{c 4-56 c 7)}$.

Comme la genèse de l'ancienne Athènes, celle du feu, de l'air, de l'eau et de la terre dans leur état cosmique a donc deux causes: l'une naturelle, l'autre artificielle. De même qu'Athéna a recueilli le rejeton né de Gê et d'Héphaïstos dans la future chōra attique, de même le démiurge a travaillé avec ce qui était né spontanément et naturellement dans la chōra précosmique, à savoir les empreintes du feu, de l'air, de l'eau et de la terre. Ensuite, il a formé ces empreintes au moyen de belles figures géométriques et ainsi engendré les quatre principaux gene de corps primaires tels que nous les percevons maintenant 
dans le cosmos, le feu, l'air, l'eau et la terre. De manière comparable, la déesse a formé le rejeton né de Gê et d'Héphaïstos en lui donnant un excellent ordre constitutionnel et a ainsi produit le meilleur et le plus beau genos humain qui ait jamais vu le jour.

Comme la genèse du genos des Athéniens, celle des quatre genē de corpuscules est racontée sans référence aucune à un modèle intelligible. En effet, l'existence du feu, de l'air, de l'eau et de la terre intelligibles semble être requise pour rendre compte de l'apparition des empreintes dans la chöra précosmique (cf. 51b6-52d4), mais non pour expliquer leur « configuration » au moyen des polyèdres. Ceux-ci sont désignés comme des éléments et des semences (cf.

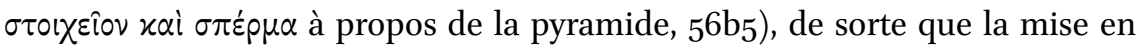
forme des empreintes s'apparente à des semailles ou à un engendrement plutôt qu'à la production d'un objet artisanal, qui se fait d'après une paradigme intelligible. Il semble en effet que les quatre genē de corpuscules soient engendrés par le dieu de manière artificielle, et non artisanale, au moyen de ces semences élémentaires dans la matrice-réceptacle et terre-mère qu'est la chōra précosmique. De fait, dans le deuxième discours on ne trouve ni le terme dēmiourgos désignant le dieu comme artisan, ni le verbe correspondant ${ }^{16}$.

Le rapprochement de la genèse des corpuscules dans la chōra précosmique avec celle de la cité d'Athènes se justifie aussi parce que les noms par lesquels

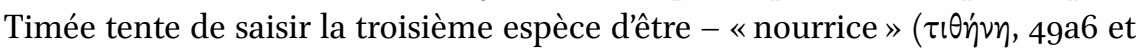

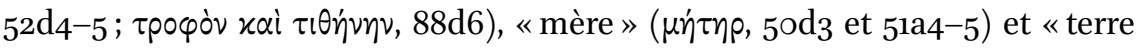

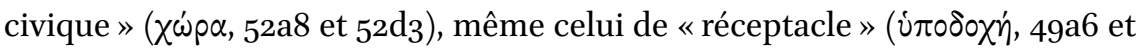
$\left.5^{1 a} 5\right)^{17}$ - appartiennent tous au champ lexical de l'imaginaire de l'autochtonie athénienne auquel faisaient précisément allusion les deux passages cités plus haut (pp. 8-9).

\section{$4 \quad$ Guerre des corpuscules, guerre des cités}

A l'instar du genos des Athéniens, les quatre genē de corps primaires sont également destinés à combattre. Chaque espèce de corpuscules occupe en effet par

16 En 59a5, dēmiourgos désigne le feu comme « artisan produisant de l'hétérogénéité » dans l'eau.

17 Le terme hypodochē peut qualifier la matrice; cf. Aristote, Gén. des anim. 4, 764b32-33. Voir aussi le participe hypodexamenē désignant la chōra-mère dans le Ménexène de Platon (237c2-3). 
nature une chōra ${ }^{18}$ et un topos ${ }^{19}$ propres dans le cosmos en raison de l'action «sismique» de la nourrice du devenir qui les sépare selon leur similitude (52d4-53a7 et $\left.57 \mathrm{~b}_{7}-\mathrm{c} 6\right)$. Toutefois, la rotation de l'univers presse les plus petits dans les interstices laissés par les plus grands $\left(58 a 2-b_{5}\right)$. Si un corpuscule pénètre dans la chōra d'un autre genos de corpuscules, soit il dissout les corpuscules qu'il heurte, soit il est lui-même dissous dans ses triangles constitutifs. A l'exception des triangles de terre, ces triangles peuvent se recomposer en un ou plusieurs corpuscules d'un autre genre ${ }^{20}$. Ces mouvements et transformations des corpuscules sont décrits dans le passage $56 \mathrm{c} 8-57 \mathrm{~b} 7$ avec un lexique guer-

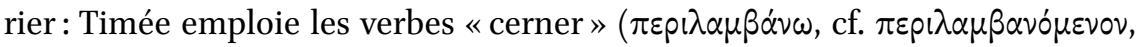

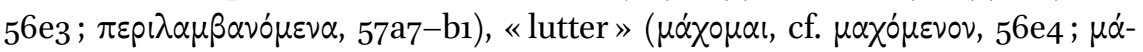

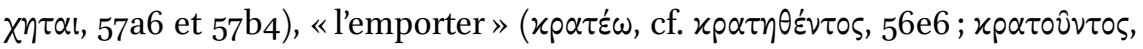

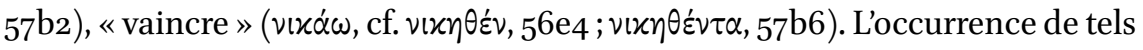
verbes permet de laisser aux notions de topos et de chōra, même dans ce contexte physique, leur signification géographique et politique courante et de les traduire par «région» et «territoire» respectivement, plutôt que par «lieu » et «place » comme l'a proposé Jean-François Pradeau ${ }^{21}$.

Tant que le cosmos accomplira sa révolution sur lui-même, les luttes entre les corpuscules ne cesseront pas en raison de la contrainte qui dicte leurs mouvements et leurs transformations. La guerre entre les corpuscules a été intégrée comme un élément constitutif du cosmos, tout comme les guerres permanentes - déclarées ou non - entre les cités font partie du monde grec ${ }^{22}$. $\mathrm{Au}$ contraire, la guerre entre l'Atlantide et l'ancienne Athènes s'est terminée par la victoire définitive de cette dernière $(25 \mathrm{~b} 5-\mathrm{c} 6)$, suivie de la disparition des deux adversaires: le corps des combattants athéniens dans la terre d'où ils sont issus, et l'île de l'Atlantide et leurs habitants dans la mer, rendant ainsi la mer extérieure inaccessible $(25 \mathrm{c} 6-\mathrm{d} 6)$. A l'époque de Solon ou de Critias le jeune, la chōra atlante n'existe plus et la chōra athénienne, on l'a déjà noté, a été érodée par de nombreuses pluies torrentielles.

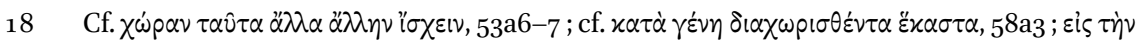

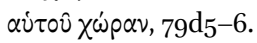

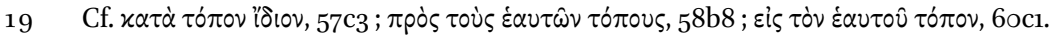

$20 \quad$ Pour les détails, voir Brisson, Platon. Timée. Critias, 301.

21 Pradeau, Le Monde de la politique, 291, distingue trois usages (physique, géographique et figuré) de topos et de chōra dans les dialogues platoniciens; selon leur usage physique «topos signifie le lieu, l'endroit où se trouve quelque chose, et chōra la place qu'occupe une chose, ou qu'elle abandonne (elle 'fait place') en se déplaçant » (italiques de l'auteur). Cf. aussi Pradeau, «Être quelque part, occuper une place. TOПО $\Sigma$ et X $2 \mathrm{PA}$ dans le Timée », 38 o et 388 ss. Pour l'usage géographique de ces deux termes, voir ci-dessus les notes 12 et 13 . 
Si l'on compare la deuxième et troisième partie du récit de Critias l'ancien dans le prologue ( $23 \mathrm{~d} 4-25 \mathrm{~d} 6$ ) au deuxième discours de Timée ( $48 \mathrm{a} 7-68 \mathrm{~d} 7$ ), on peut remarquer que Critias évoque une évolution du monde inverse de celle de Timée. Cela se reflète dans l'ordre dont chaque phase est traitée dans le discours (cf. les plans des discours donnés ci-dessus p. 6-7 et 12): Timée décrit d'abord la chōra précosmique, cette troisième espèce d'être difficile et obscure, qu'il est forcé d'introduire pour expliquer la perception sensorielle et dont

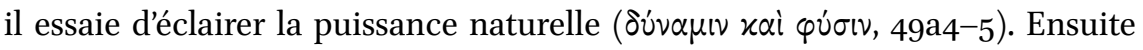
seulement, il peut expliquer comment y naissent, grâce à l'œuvre ordonnatrice du démiurge, à partir des empreintes désordonnées, les quatre genē de corpuscules (et leurs nombreuses espèces) que nous percevons maintenant dans le cosmos. Au contraire, Critias commence par la naissance et l'organisation politique du genos des anciens Athéniens. Elles ont eu lieu dans un temps antédiluvien, où la chōra attique était encore intacte et belle (cf. Critias 11od4111e5) et l'espace marin, situé au-delà des colonnes d'Hercule, toujours ouvert à la navigation. Ce n'est que tout à la fin de son récit que fait irruption, à la fois dans son discours et dans le monde civilisé et ordonné de l'oikoumenē la

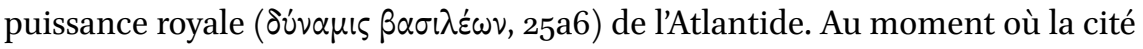
insulaire commence la guerre, son immense territoire a subi de nombreuses transformations et fait plutôt penser à la chōra précosmique où « tout cela se

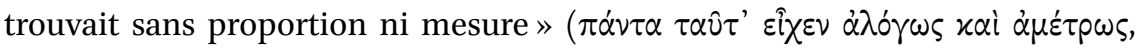
53a8). Neuf millénaires plus tard, la terre civique des Athéniens se retrouve dans le même état. Quand Critias parle, elle ne présente plus que des restes de sa fertilité et de sa splendeur passées. Elle aussi ressemble désormais à la chōra précosmique, «lorsqu'un dieu en est absent » (53b3-4).

\section{Le statut de vérité de l'ancien discours de Critias le jeune}

Pour répondre au vœu de Socrate et mettre la meilleure cité en mouvement sinon dans les faits, du moins dans un discours, la notion d'alētheia, « vérité » et « réalité », joue un rôle aussi important que celles de genos et de chōra. Il se pourrait bien qu'il y ait un rapport entre la vérité du discours et ses aspects généalogiques et « chôrologiques ».

La vérité $\mathrm{du}$ « discours certes très étrange et pourtant tout à fait vrai » ( $\lambda$ ó you

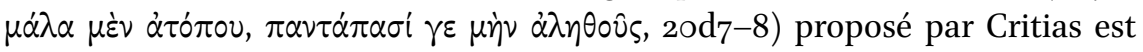
étroitement liée à son parcours dans le temps généalogique et dans l'espace géographique et politique. D'une part, Critias le jeune tient ce discours de son grand-père homonyme, Critias l'ancien, qui l'avait lui-même entendu de Solon, un parent et ami de Drôpidès, son arrière-grand-père (2od7-21a3). 
La transmission continue de ce logos d'une génération à l'autre au sein de la famille ( $\gamma^{\varepsilon} v \circ \varsigma$ ) de Critias le jeune ${ }^{23}$ semble, du moins pour ce dernier, une garantie de sa vérité. Ajoutons que les citoyens de Saïs, située dans le delta du Nil, où Solon a appris ce récit, sont eux-mêmes en quelque sorte apparentés aux anciens Athéniens, car leur cité a été fondée par la déesse Athéna sous le nom de Neïth (21e1-7, cf. 23d4-e2). D'autre part, ce discours "parfaitement vrai » provient d'Egypte, terre $(\chi \omega \dot{p} \alpha)$ qui n'est jamais ravagée par les pluies diluviennes et qui est protégée des incendies par les crues du Nil $\left(22 \mathrm{~d}_{5}-6\right.$ et e2-4). C'est un pays où rien n'est jamais détruit et tout reste intact, un pays de lettrés où rien ne s'oublie et tout se conserve, un pays de vieillards où les gens ne rajeunissent jamais dans leur âme. C'est là que sont documentés et archivés depuis des lustres tous les événements et tous les savoirs extraordinaires du monde (22e4-23a5), si bien que le discours d'un très vieux prêtre qui vit dans un tel territoire ne saurait être «un récit d'enfants » $\left(\pi \alpha i \delta \omega \nu \mu \hat{v} \theta_{0 \varsigma}\right)$, mais seulement un « discours vrai » ( $\left.\lambda \circ \gamma_{0} \circ \alpha_{\alpha} \lambda \eta \theta^{\prime} \varsigma\right)$. Le long récit de l'origine généalogique et géographique de ce logos est donc essentiel pour la véracité que Critias cherche à lui conférer.

Voyons maintenant brièvement quels rôles jouent les notions de chōra et de genos pour la transposition ( $\mu \varepsilon \tau \alpha \varphi \circ \rho \alpha \alpha$ ) de la meilleure cité dans la réalité ( $\dot{\varepsilon} \pi i \tau \dot{\alpha} \lambda \eta \theta \hat{\varepsilon} \varsigma, 26 \mathrm{~d} \mathbf{1})$. Après avoir résumé le contenu du discours du prêtre égyptien, Critias propose à Socrate de transposer la meilleure cité, élaborée en pa-

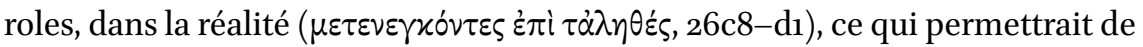
répondre parfaitement à son vœu :

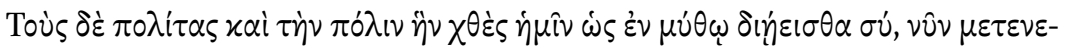

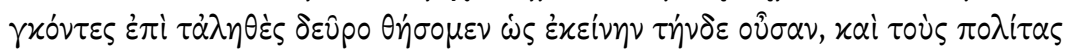

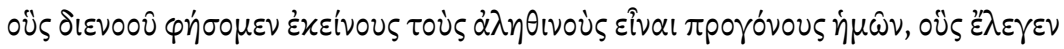

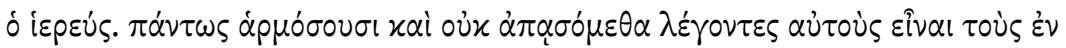

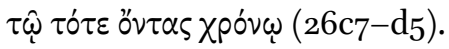

Les citoyens et la cité que, toi, tu nous décrivais hier comme dans un récit ( $\dot{\omega} \varsigma \dot{\varepsilon} \nu \mu \dot{v} \theta \omega)$, en les transposant maintenant ici dans le réel ( $\dot{\varepsilon} \pi i \tau \dot{\alpha} \lambda \eta \theta \theta \dot{\varepsilon} \varsigma)$, nous allons faire comme si celle-là était celle-ci, et les citoyens que tu as imaginés, nous prétendrons que ce sont nos vrais ancêtres, ceux dont parlait le prêtre. Ils concorderont entièrement et nous ne détonnerons pas en disant qu'ils sont ceux qui existaient en ce temps-là.

23 Platon lui-même appartenait à cette famille : sa mère, Périctionè, était la fille de Glaucon, un oncle de Critias le tyran, que j'identifie avec Critias le jeune ; cf. Brisson, Platon. Timée. Critias, 328 . 


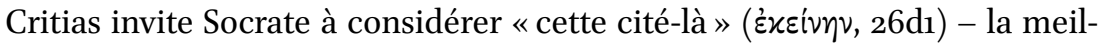
leure cité - comme identique avec «cette cité-ci» ( $\tau \dot{\eta} v \delta \varepsilon, 26 \mathrm{~d} 1)$ - l'Athènes

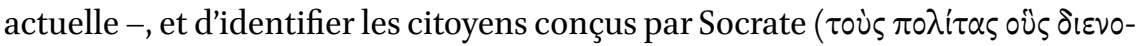

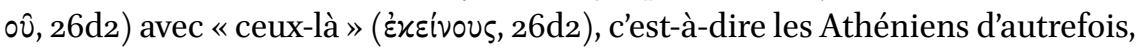

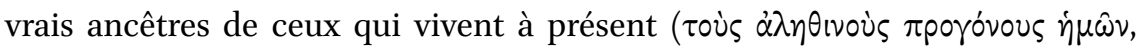
$26 \mathrm{~d} 2-3)$.

La transposition dans la réalité s'opère donc sur deux plans, spatial et temporel. Du point de vue spatial, la meilleure cité sera localisée à Athènes même, là où se déroule le dialogue entre Socrate, Critias, Timée et Hermocrate. D'un point de vue temporel, les habitants de la meilleure cité seront projetés dans un temps très reculé et identifiés avec les lointains ancêtres des Athéniens actuels, dont Socrate et Critias ici présents. La cité sera donc inscrite non pas dans le hic et nunc, mais dans le hic et tunc, c'est-à-dire transposée ici même dans la chōra d'Athènes et en même temps identifiée du point de vue généalogique avec un genos athénien d'un passé fort lointain. Ou bien, comme le dit Critias un peu plus tard, il s'agit de «faire [des citoyens-gardiens de la meilleure cité de Socrate] des citoyens de cette cité-ci, puisqu'ils sont les

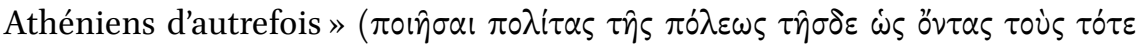
'A $\eta$ nvaious, 27b2-3). L'identification de la meilleure cité à l'ancienne Athènes et la transposition dans la réalité qu'elle implique font d'elle une cité dont on peut dire où, quand et de quels parents elle est née, une cité qui peut être située d'un point de vue tant «chôrologique » que généalogique. En outre, cela en fait une cité qui n'existe pas seulement dans mais aussi en dehors du discours. A la différence de l'Atlantide, engloutie à tout jamais dans l'Océan et confinée dorénavant à sa seule existence intradiscursive, la cité d'Athènes a une double existence, intra- et extradiscursive. Dès lors, l'identification de la meilleure cité avec l'ancienne Athènes que Critias propose à Socrate signifie pour elle davantage qu'une simple transposition dans un espace et un temps intradiscursifs : elle l'inscrit également dans un territoire et un temps généalogique extradiscursifs. La meilleure cité est ainsi doublement transposée dans la réalité, celle du logos alēthēs rapporté d'Egypte par Solon et celle de l'Athènes du temps de Critias le jeune, et elle est mise en rapport tant avec le passé qu'avec le présent.

Comme certains lecteurs modernes, Critias comprend donc le vœu de Socrate comme un souhait de voir sa cité réalisée dans l'« Histoire », d'entendre un discours qui aille au-delà de la mimēsis fictionnelle d'un récit de guerre ${ }^{24}$.

24 Cf. p. ex. Hadot, «Physique et poésie dans le Timée de Platon », 115 : «Il [Socrate] aimerait bien qu'on lui montre sa Cité idéale cette fois en action, autrement dit qu'on la retrouve dans l'Histoire»; Detienne, L'écriture d'Orphée, 169-170: «Socrate voudrait qu'on lui 
Si le discours de Critias est «vrai » ( $\left.\dot{\alpha} \lambda \eta \theta^{\prime} \varsigma\right)$ par opposition à ce que Socrate a raconté «comme dans un récit» ( $\dot{\omega} \varsigma \dot{\varepsilon} \nu \mu \dot{v} \theta \omega)$, s'il ne s'agit justement pas d'un récit façonné ( $\mu \hat{v} \theta 0 \varsigma \pi \lambda \alpha \sigma \theta \varepsilon i \varsigma$, cf. 26e4), c'est du fait qu'il est un discours « chôrologique » et généalogique qui enracine la cité dans un territoire propre et la situe dans le temps humain à la fois dans et en dehors du discours. Une fois une chōra et une généalogie humaine intégrées dans le récit de Socrate, celui-ci se transforme en un logos alēthès.

Timée, quant à lui, est moins affirmatif : pour son discours cosmogonique et anthropogonique, il n’aspire qu'à la vraisemblance. Son eikōs logos, « discours vraisemblable» - qu'il appelle aussi eikōs mythos, « récit vraisemblable » ${ }^{25}$ occupe une place au (juste) milieu entre le récit de Socrate et le discours vrai de Critias. Il n'empêche que l'intégration de la chōra et des genè de corpuscules augmente aussi la vérité de son discours. Pour son deuxième discours, Timée revendique en effet davantage de vraisemblance que pour le premier (cf. $\mu \eta$ -

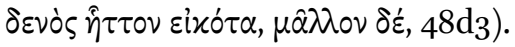

A cet égard, son premier discours, qui raconte l'œuvre de l'intellect, se rapproche du récit «idéaliste» de Socrate, récapitulé au début du prologue. Le deuxième discours, par contre, qui décrit l'œuvre de la contrainte, fait écho au discours « réaliste» de Critias. En d'autres termes, d'un point de vue narratif, le monde où sont nées l'ancienne Athènes et l'Atlantide et où a eu lieu la guerre qui les opposait, évoqué dans le récit du prêtre égyptien, prépare celui dans lequel sont nés les différents genres de corpuscules dont les mouvements obéissent à la contrainte. C'est un monde où les dieux, certes, ne sont pas absents mais où ils n'œuvrent pas comme artisans ${ }^{26}$.

montre, en projection privée, la cité idéale telle quelle, mais en action, en branle. [...] Plus précisément, que pourraient être les exploits, les áthloi, de la cité idéale ? Que devient la Kallípolis jetée dans les eaux rapides de l'Histoire?». Pour un aperçu de la discussion autour du statut de vérité et du genre littéraire du récit de l'Atlantide dans le Timée-Critias, on peut se référer à Brisson, Platon. Timée. Critias, 313-325; Gill, Plato : The Atlantis Story, (introduction), et Gill, Plato's Atlantis Story: Text, Translation and Commentary, 1-48; Pradeau, Le Monde de la politique, 66-110; Vidal-Naquet, L'Atlantide. Petite histoire d'un mythe platonicien.

25 En comparaison des nombreuses occurrences de l'expression eikōs logos, eikōs mythos n'apparaît que trois fois $(29 \mathrm{~d} 2,59 \mathrm{c} 6$ et $68 \mathrm{~d} 2)$ dans le discours de Timée.

26 Je remercie Jakub Jirsa, Filip Karfík, Štěpán Špinka de m’avoir invitée à présenter ces réflexions sur le prologue du Timée-Critias au dixième Symposium Platonicum Pragense, organisé par leurs soins en novembre 2015. Un grand merci aussi à Kelly Harrison pour sa relecture attentive. 


\section{Works Cited}

Bonnard, Jean-Baptiste. Le Complexe de Zeus. Représentations de la paternité en Grèce ancienne. Paris : Publications de la Sorbonne, 2004.

Brisson, Luc. Platon. Timée. Critias. Paris : Flammarion, 1992, quatrième édition 1999.

Brisson, Luc. «Platon face à la guerre». Cahiers d'Etudes Lévinassiennes 14 (2016): 34-44.

Cambiano, Giuseppe. «La pace in Platone e in Aristotele ». Dans La Pace nel mondo antico (Atti del Convegno Nazionale di Studi, Torino 9-10-11 Aprile 1990). Sous la direction de Renato Uglione. Torino : Associazione Italiana di Cultura Classica, 1991, 97-114.

Campese, Silvia et Lucia Loredana Canino. «La genesi della polis». Dans Platone. La Repubblica. Sous la direction de Mario Vegetti. Napoli : Bibliopolis, 1998-2007, Vol. II, 285-332.

Casevitz, Michel. Le vocabulaire de la colonisation en grec ancien. Etude lexicologique:

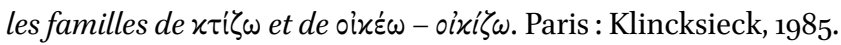

Detienne, Marcel. L'écriture d'Orphée. Paris : Gallimard, 1989.

Fowler, R. L. «Genealogical Thinking, Hesiod's Catalogue, and the Creation of the Hellenes ». Proceedings of the Cambridge Philological Society 44 (1998):1-19.

Georgoudi, Stella. «Gaia/Gê. Entre mythe, culte et idéologie ». Dans Myth and Symbol I: Symbolic phenomena in ancient Greek culture (Papers from the first international symposium on symbolism at the University of Tromsø, June $4-7,1998)$. Sous la direction de Synnøve des Bouvrie. Bergen: The Norwegian Institute of Athens, 2002, $113-134$.

Gill, Christopher. Plato : The Atlantis Story. Bristol : Classical Press, 1980.

Gill, Christopher. Plato's Atlantis Story: Text, Translation and Commentary. Liverpool: University Press, 2017.

Graf, Fritz. «Zwischen Autochthonie und Immigration : Die Herkunft von Völkern in der Alten Welt». Dans Anfänge. Sous la direction de Detlev Clemens et Tilo Schabert. München : W. Fink, 1998, 65-93.

Hadot, Pierre. «Physique et poésie dans le Timée de Platon ». Revue de Théologie et de Philosophie 115 (1983) : 113-133.

Jouanna, Jacques, éd. et trad. Hippocrate. Tome II, $2^{\mathrm{e}}$ partie: Airs, Eaux, Lieux. Paris : Les Belles Lettres, 1996.

Loraux, Nicole. Les enfants d'Athéna. Idées athéniennes sur la citoyenneté et la division des sexes. Deuxième édition. Paris : Seuil, 1990.

Parker, Robert. « Myths of Early Athens ». Dans Interpretation of Greek Mythology. Sous la direction de Jan Bremmer. London : Croom Helm, 1987, 187-214.

Pradeau, Jean-François. « Être quelque part, occuper une place. TOПО $\Sigma$ et X $\Omega$ PA dans le Timée ». Les études philosophiques 1 (1995) : 375-399. 
Pradeau, Jean-François. Le Monde de la politique. Sur le récit atlante de Platon, Timée (17-27) et Critias. Sankt Augustin : Academia, 1997.

Rivaud, Albert. Platon. OEuvres complètes. Tome x. Timée-Critias. Paris: Les Belles Lettres, 1925.

Vidal-Naquet, Pierre. Le chasseur noir. Formes de pensée et formes de société dans le monde grec. Troisième édition. Paris : La Découverte, 2005.

Vidal-Naquet, Pierre. L'Atlantide. Petite histoire d'un mythe platonicien. Paris : Les Belles Lettres, 2005 . 